\title{
Effect of anharmonicities in the critical number of trapped condensed atoms with an attractive two-body interaction
}

\author{
Victo S. Filho, ${ }^{1}$ Arnaldo Gammal, ${ }^{1,2}$ and Lauro Tomio ${ }^{1}$ \\ ${ }^{1}$ Instituto de Física Teórica, Universidade Estadual Paulista, 01405-900 São Paulo, Brazil \\ ${ }^{2}$ Instituto de Física, Universidade de São Paulo, 05315-970 São Paulo, Brazil
}

(Received 1 March 2002; revised manuscript received 21 June 2002; published 10 October 2002)

\begin{abstract}
We determine the quantitative effect, in the maximum number of particles and other static observables, due to small anharmonic terms added to the confining potential of an atomic condensed system with negative two-body interaction. As an example of how a cubic or quartic anharmonic term can affect the maximum number of particles, we consider the trap parameters and the results given by Roberts et al. [Phys. Rev. Lett. 86, 4211 (2001)]. However, this study can be easily transferred to other trap geometries to estimate anharmonic effects.
\end{abstract}

DOI: 10.1103/PhysRevA.66.043605

\section{INTRODUCTION}

The experimental realization of Bose-Einstein condensation (BEC) in magnetically trapped weakly interacting atoms [1-4] brought a lot of interest in theoretical studies of the properties of condensed atomic systems. For systems with negative two-body scattering length, recently it was reported in Ref. [5] a discrepancy between experimental results and theoretical predictions [6], in the maximum critical number $N_{c}$ of trapped atoms. In Ref. [7], it was shown that part of the discrepancy can be explained by taking into consideration the axially deformed shape of the trap. The theoretical prediction of the critical number of atoms, for the cylindrical symmetry considered in Ref. [5], has to be adjusted to a number that is lower than the number given by the corresponding spherically symmetric trap. Still, this correction is not enough to obtain a result that is totally compatible with experimental values. So, it is relevant to look for other possible sources of the observed discrepancy, in this specific case; as for example, higher-order nonlinearities in the meanfield approximation [8], or possible experimental deviations not already taken into account.

The parameter associated with the critical number $N_{c}$, given in Ref. [5], is defined by

$$
k=\frac{N_{c}|a|}{\sqrt{\hbar /(m \omega)}},
$$

where $\omega \equiv\left(\omega_{x} \omega_{y} \omega_{z}\right)^{1 / 3}$ is the geometrical mean value of the trap frequencies, $m$ the mass of the atomic species, and $a$ is the two-body scattering length of the particles in the condensate. With the assumption of a spherical symmetrical trap, $k \cong 0.575$ [6]. The critical number of atoms for Bose-Einstein condensates with $a<0$ have been investigated by the JILA group, taking into account experiments with ${ }^{85} \mathrm{Rb}$ [5], considering a wide tuning of the scattering length $a$, from positive to negative values, by means of Feshbach resonance [9]. The experimental result for the number $k, k_{\text {expt }}=0.459$ \pm 0.012 (statistical) \pm 0.054 (systematic), corresponds to a deviation of about $20 \%$ lower than the predicted spherical symmetrical result. Part of this discrepancy was shown to be related to the cylindrical cigar-shaped symmetry employed in
PACS number(s): 03.75.Fi, 32.80.Pj, 05.30.Jp

the JILA's experiment, where the frequencies corresponding to the directions $z$ and $r_{\perp} \equiv \sqrt{x^{2}+y^{2}}$ are, respectively, given by $\omega_{z}=2 \pi \times 6.8 \mathrm{~Hz}$ and $\omega_{\perp}=2 \pi \times 17.35 \mathrm{~Hz}$. So, the parameter of anisotropy is given by $\lambda \equiv \omega_{z} / \omega_{\perp}=0.3919$. The theoretical prediction, obtained in Ref. [7], with the correct cylindrical symmetry given in Ref. [5], is $k \cong 0.55$. In this case, the experimental result is smaller than the predicted value by about $16.5 \%$. Such a result is still not enough to include the theoretical result within the error bars of the experimental result.

As explained in Ref. [10], in the JILA's experiments with ${ }^{85} \mathrm{Rb}$, the trap is practically harmonic in the central region, for very low temperatures and for small-size condensates. However, one should check how a deviation of the harmonic trap, outside the central region, can affect the number of condensed atoms. In this case, we are considering the possible existence of imprecisions in the form of the confining potential, generated by the modified Ioffe-Pritchard design used (known as baseball trap). The modification of the ground-state solution of the condensed state can alter, correspondingly, the observables associated with it.

In our present investigation we consider a deviation in the harmonic trap potential that is effective only outside the central part of the potential, with the addition of a term that is proportional to a cubic or quartic power of the distance. This work was first motivated by looking for a possible source of the observed discrepancy between theory and the JILA's experiment. However, we realize that, if any deviation exists from the harmonic trap in the experiments reported in Ref. [5], it should be of a very small factor considering the kind of trap design used by them [10]. But, one should be aware also of other kind of trap arrangements in the experimental studies of BEC, where an investigation of possible effects in the observables due to anharmonic terms in the interaction can be useful. In this perspective, our present study of the effect of anharmonic terms added to a harmonic trap interaction, is not restricted to the example that we are going to consider. We consider the trap parameters of the JILA's experiments [5] as an example, estimating deviations in $N_{c}$ due to anharmonicities, which can easily be extended to other geometrical trap configurations, with the help of previous studies [7]. Presently, we are reporting on the numerical results obtained for $N_{c}$ when the trap deviates from the har- 
monic shape. We should also note that there exists a previous study considering the occurrence of anharmonic terms in a time orbiting potential trap [11].

As shown by our results, a steeper confinement will result in a lower $k$, against the naive expectation. At first sight, a steeper confinement should increase the kinetic and potential energies, with stabilization for a larger (and not smaller) value of $k$. However, a steeper confinement also means that the relative distance between the atoms is less than in the case of the harmonic potential. Therefore, with the atoms experiencing more attraction from the interaction term, this will result in collapse for smaller $k$.

Next, we review the formalism used in the present approach, followed by the main results and conclusions.

\section{MEAN-FIELD APPROXIMATION}

The Gross-Pitaevskii equation that describes the wave function of the condensate $\Psi$ in the mean-field approximation has the form

$$
i \hbar \frac{\partial \Psi}{\partial t}=\left(-\frac{\hbar^{2}}{2 m} \vec{\nabla}^{2}+U_{\text {trap }}-\frac{4 \pi \hbar^{2}|a|}{m}|\Psi|^{2}\right) \Psi,
$$

where the potential $U_{\text {trap }} \equiv U_{\text {trap }}\left(\vec{r} ; \omega_{x}, \omega_{y}, \omega_{z}\right)$ is given by a modified harmonic oscillator trap, and the wave function $\Psi$ $\equiv \Psi(\vec{r}, t)$ is normalized to the number of atoms, $N$. For the stationary solution of Eq. (2), with $\Psi(\vec{r}, t)$ $=\exp (-\mathrm{i} \mu t / \hbar) \Psi(\vec{r}, 0)$, where $\mu$ is the chemical potential, we obtain

$$
i \hbar \frac{\partial \Psi}{\partial t}=\mu \Psi
$$

Considering the symmetry used in the JILA's experiment, where the trap frequencies are given by $\omega_{\perp} \equiv \omega_{x}=\omega_{y} \neq \omega_{z}$, it is adequate to work in cylindrical coordinates:

$$
r_{\perp}=\sqrt{x^{2}+y^{2}} \quad \text { and } \quad \theta=\arctan \left(\frac{y}{x}\right) .
$$

For the ground state of the condensate, we have $\Psi$ $\equiv \Psi\left(r_{\perp}, z, t\right)$. By using the trapping geometrical average frequency $\omega \equiv\left(\omega_{x} \omega_{y} \omega_{z}\right)^{1 / 3}$ and the oscillator length $l_{o}$ $\equiv \sqrt{\hbar /(m \omega)}$, we obtain the following dimensionless coordinates and parameters:

$$
\begin{gathered}
\rho \equiv \sqrt{2} \frac{r_{\perp}}{l_{o}}, \quad \xi \equiv \sqrt{2} \frac{z}{l_{o}}, \tau \equiv \omega t, \\
\omega_{\rho} \equiv \frac{\omega_{\perp}}{\omega}, \quad \omega_{\zeta} \equiv \frac{\omega_{z}}{\omega} .
\end{gathered}
$$

With the above dimensionless units, redefining $\Psi$,

$$
\Phi(\rho, \zeta, \tau) \equiv \sqrt{\frac{4 \pi \hbar|a|}{m \omega}} \Psi(\vec{r}, t),
$$

and the trap potential $U_{t r a p} \equiv \hbar \omega V(\rho, \zeta)$, Eq. (2) can be rewritten as

$$
i \frac{\partial \Phi}{\partial \tau}=\left[-\frac{1}{\rho} \frac{\partial}{\partial \rho}\left(\rho \frac{\partial}{\partial \rho}\right)-\frac{\partial^{2}}{\partial \zeta^{2}}+V(\rho, \zeta)-|\Phi|^{2}\right] \Phi,
$$

where the normalization of $\Phi$ is given by

$$
\int_{-\infty}^{\infty} d \zeta \int_{0}^{\infty} d \rho \rho|\Phi|^{2}=4 \sqrt{2} \frac{N|a|}{l_{0}} \equiv 2 n .
$$

In the critical limit, we have $n=n_{c}=2 \sqrt{2} k$.

The equation, corresponding to Eq. (3), for the chemical potential $\beta$ in dimensionless units $(\mu=\hbar \omega \beta)$ is given by

$$
\left[-\frac{1}{\rho} \frac{\partial}{\partial \rho}\left(\rho \frac{\partial}{\partial \rho}\right)-\frac{\partial^{2}}{\partial \zeta^{2}}+V(\rho, \zeta)-|\Phi|^{2}\right] \Phi=\beta \Phi .
$$

As is known [12], Eq. (9) is valid in the mean-field approximation of the quantum many-body problem of a dilute gas, where the average interparticle distances are much larger than the absolute value of the scattering length, and the wavelengths are much larger than the average interparticle distance.

The total energy of the system is given by

$$
\begin{aligned}
E= & \frac{N \hbar \omega}{2 n} \int_{0}^{\infty} \rho d \rho \int_{-\infty}^{\infty} d \zeta\left\{\left[\frac{\partial}{\partial \rho} \Phi\right]^{2}+\left[\frac{\partial}{\partial z} \Phi\right]^{2}\right. \\
& \left.+V(\rho, \zeta)|\Phi|^{2}-\frac{|\Phi|^{4}}{2}\right\} .
\end{aligned}
$$

In order to analyze the effect of a deviation of the trap potential from the harmonic behavior, we consider two expressions for $V(\rho, \zeta)$, labeled by $\nu=1,2$, as follows:

$$
V^{(\nu)}(\rho, \zeta)=\frac{1}{4}\left[\omega_{\rho}^{2} \rho^{2}\left(1+\delta_{\rho} \rho^{\nu}\right)+\omega_{\zeta}^{2} \zeta^{2}\left(1+\delta_{\zeta} \zeta^{\nu}\right)\right] .
$$

The distortion added to the harmonic potential is cubic when $\nu=1$ and quartic when $\nu=2$. In both cases, the magnitudes of the distortions in the directions $\rho$ and $\zeta$ are given by the parameters $\delta_{\rho}$ and $\delta_{\zeta}$.

With Eq. (11), in both cases one can observe that the interaction is approximately harmonic near the center of the trap (at $\rho=0$ and/or $\zeta=0$ ). When $\rho<1$, the quartic term keeps approximately the harmonic shape of the potential in a more effective way than the cubic term. In Fig. 1, we can see how the shape of the harmonic trap $\left(\delta_{\rho}=0\right)$ changes in the radial direction, at the position $\zeta=0$, when one adds a cubic $(\nu=1)$ or a quartic $(\nu=2)$ term in the potential.

\section{RESULTS}

In this section, we report on our main results considering the solutions for the chemical potential, obtained for Eq. (9) with the anharmonic interaction given by Eq. (11), for several combinations of the parameters $\delta_{\rho}$ and $\delta_{\zeta}$, for $\nu=1$ and 


\section{$\mathbf{V}(\rho, \zeta)$}

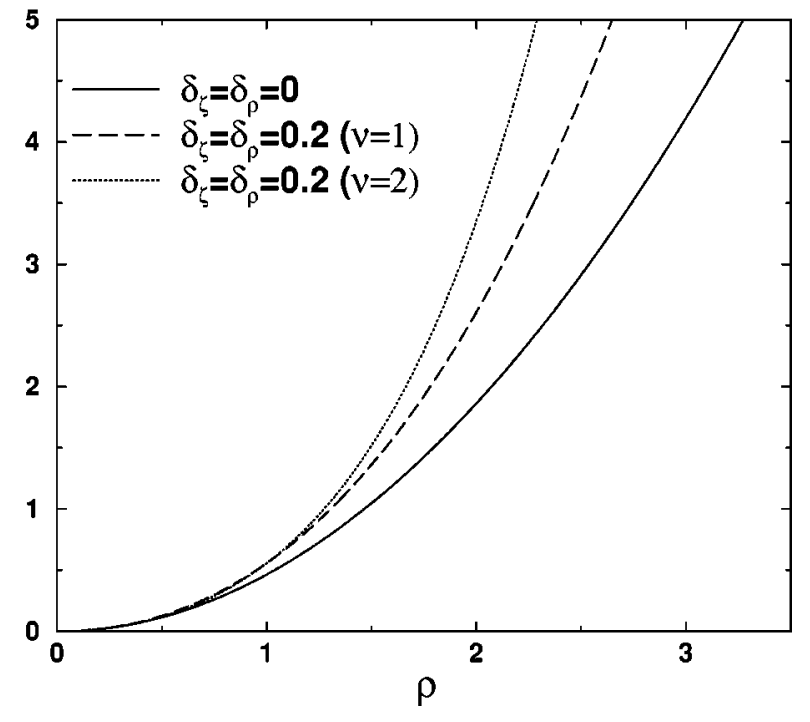

FIG. 1. The anharmonic potentials, as given in Eq. (11), with $\delta_{\rho}=0.2$ and $\zeta=0$, for the cubic ( $\nu=1$, dashed line) and quartic $(\nu=2$, dotted line) cases, are compared with the harmonic potential ( $\delta_{\rho}=0$, solid line). All the units are dimensionless, such that $U_{\text {trap }}=(\hbar \omega) V$ and $r_{\perp}=\rho l_{o} / \sqrt{2}$. The values of the frequencies correspond to the ones given in Ref. [5]: $\omega_{z}=2 \pi \times 6.8 \mathrm{~Hz}$ and $\omega_{\perp}$ $=2 \pi \times 17.35 \mathrm{~Hz}$.

$\nu=2$. We have also considered the solutions for the total energy, which is given by Eq. (10). For the numerical solution of Eq. (9), which is given in cylindrical symmetry, we have employed the Crank-Nicolson algorithm in two dimensions, employing the relaxation method propagating in the imaginary time, as earlier described in Ref. [7]. The spatial discretization used was $140 \times 140$, with cutoff $\rho_{\max }=7$ and $\zeta_{\max }=7$. The time discretization was $\Delta \tau=0.005$, and the relaxation time used for obtaining the solutions of stationary ground state was $\tau=16$. The results are more sensitive to the spatial grid spacing, but a lack of precision occurs in the third decimal figure of the values obtained in the numerical simulations. With these grids, we obtain precise results up to the second decimal figure, confirmed by the convergence obtained with a refined spatial grid $(160 \times 160)$. We obtain our results for the total energy and the chemical potential of the condensate, as functions of the number of particles, by considering the geometry of the trap given in JILA's experiment reported in Ref. [5], where $\omega_{z}=2 \pi \times 6.8 \mathrm{~Hz}$ and $\omega_{\perp}=2 \pi$ $\times 17.35 \mathrm{~Hz}$. We consider a range of values for the parameters $\delta_{\rho}$ and $\delta_{\zeta}$, which represents the corresponding anharmonicity. As shown, only positive anharmonic terms added to the original harmonic trap can make the theoretical prediction for the maximum critical number $k$ become closer to the JILA's experimental result. This implies that one should check for possible deviation of the harmonic potential, outside the central region, which makes the trap more confining.

In Table I, we present our results for the maximum critical number $k$, which is related to the critical number $N_{c}$ of atoms, as given by Eq. (1), considering several possible choices for the anharmonic parameters $\delta_{\rho}$ and $\delta_{\zeta}$, for both
TABLE I. Values of the maximum critical number $k$, for several combinations of the parameters $\delta_{\rho}$ and $\delta_{\zeta}$, related to the anharmonic factors introduced in the interaction, as given in Eq. (11), for $\nu=1$ (cubic) and $\nu=2$ (quartic). The cylindrical symmetry is the same as that given in Ref. [5].

\begin{tabular}{cccc}
\hline \hline$\nu$ & $\delta_{\rho}$ & $\delta_{\zeta}$ & $k$ \\
\hline 1 & 0.0 & 0.0 & 0.550 \\
1 & 0.1 & 0.1 & 0.529 \\
1 & 0.1 & 0.0 & 0.532 \\
1 & 0.2 & 0.2 & 0.512 \\
1 & 0.5 & 0.5 & 0.476 \\
2 & 0.1 & 0.1 & 0.520 \\
2 & 0.1 & 0.0 & 0.525 \\
2 & 0.2 & 0.2 & 0.497 \\
2 & 0.5 & 0.5 & 0.456 \\
\hline \hline
\end{tabular}

cases $\nu=1$ (cubic) and $\nu=2$ (quartic).

In Fig. 2, we show some results for the case with $\nu=2$, where we have a quartic anharmonic term added to the original interaction, in both directions $\rho$ and $\zeta$, such that $\delta_{\rho}$ $=\delta_{\zeta}$. In this example, we are also considering the cigarlike symmetry of the trap used in Ref. [5], with $\omega_{\perp} / \omega_{z}$ $=17.35 / 6.8$. We present two frames: for the total energy $E$ (lower frame), given in units of $(N \hbar \omega) /(2 n)$ and for the corresponding chemical potential $\mu$ (upper frame), in units of $\hbar \omega$, versus $N|a| / l_{o}$.

This picture does not change too much when we switch

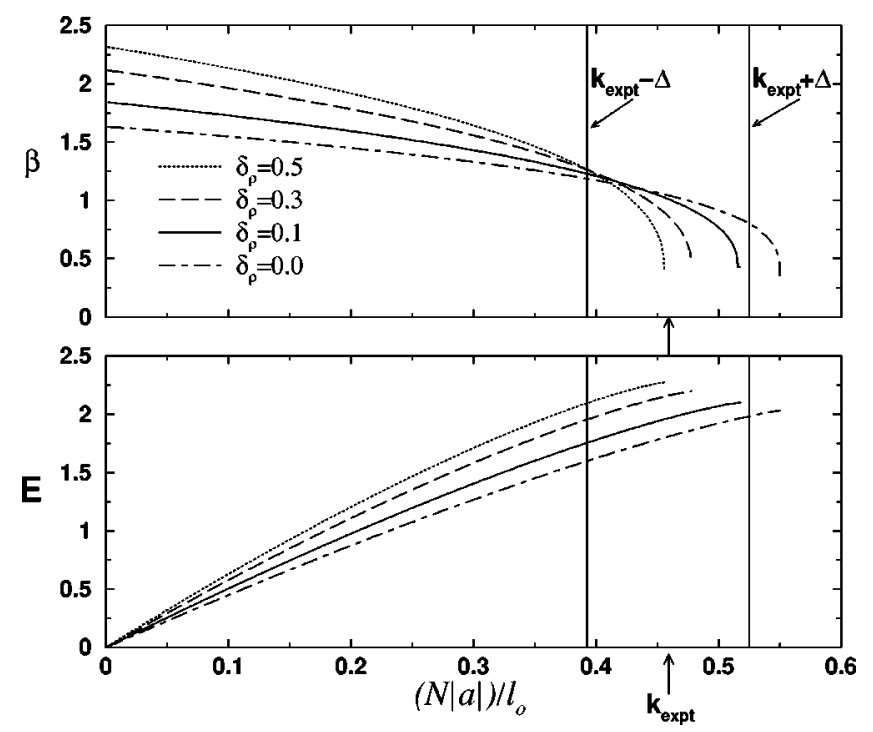

FIG. 2. Total energies $E$ (lower frame), in units of $(N \hbar \omega) /(2 n)$ $\left[E_{\text {total }} \equiv E(N \hbar \omega) /(2 n)\right]$, and chemical potentials $\mu$ (upper frame), in units of $\hbar \omega[\mu \equiv \beta(\hbar \omega)]$, versus $N|a| / l_{o}$, for different values of $\delta_{\rho}=\delta_{\zeta}$, as given inside the upper frame. The deviations from the harmonic trap are given by quartic terms in both directions $\rho$ and $\zeta$. Here, we are also using the trap considered in Ref. [5], with the asymmetry given by $\omega_{\perp} / \omega_{z}=2.5515$. The corresponding critical numbers are at the end of each curve, with the position of the experimental value $k_{\text {expt }}$ pointed out with vertical arrows. $\Delta$ corresponds to the sum of the experimental errors. 


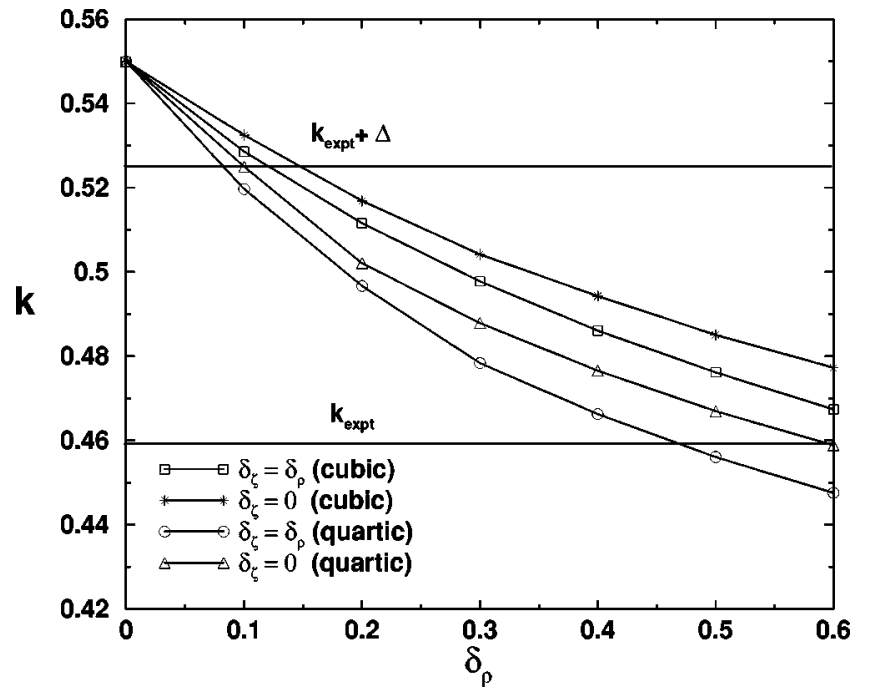

FIG. 3. Variations of the critical number of atoms $(k)$, in the condensate as functions of $\delta_{\rho}$. The corresponding deviations in the $\zeta$ direction, $\delta_{\zeta}$, are given inside the figure. The position of the experimental $k$ and the corresponding positive error bar $(\Delta)$ are also indicated.

off the deviation in the $\zeta$ direction, with $\delta_{\zeta}=0$ (the trap remains harmonic in the $z$ direction). For the case with $\nu$ $=1$, where we consider cubic anharmonic terms added to the original interaction, our results show that for both $\delta_{z}=\delta_{\rho}$ and $\delta_{\zeta}=0$ there are no significant qualitative differences in the observables plotted, and the values are approximately equal to the quartic case. The behavior is similar for all the cases considered, with the collapse of the condensate occurring for smaller critical number as we add larger positive deviation in the harmonic trap. In this respect, the addition of a quartic term $(\nu=2)$ in the potential is more effective in keeping the harmonic shape near the center of the trap.

All the above results can be globally analyzed by examining Fig. 3, in which we have the behavior of the maximum critical number of atoms in the condensate state, parametrized by $k$, as a function of the magnitude of the trapping anharmonic potential, parametrized by $\delta_{\rho}$. We display the effect of both cubic and quartic anharmonicities in confinement, with or without including the deviation in the $z$ direction. As we can see, the higher the anharmonic parameter, the smaller the critical number of condensed atoms. As one can observe, in order to obtain the theoretical results for $k$ inside the region covered by the experimental error bars, one needs deviations of about (10-20)\% from the harmonic trap, at distances of the order of the oscillator length.

\section{CONCLUSIONS}

In summary, we have solved numerically the stationary Gross-Pitaevskii equation in cylindrical symmetry, for condensed systems with attractive two-body interaction, with anharmonic trapping potentials, either with cubic and quartic deviation from the harmonic oscillator. We did the present study in the perspective to observe the real effect on $N_{c}$ of anharmonicities that can occur in the usual confining traps that have been extensively employed in the experiments with atomic BEC. We took the harmonic trap parameters considered in Ref. [5], to exemplify how much should be the strength of cubic or quartic anharmonic terms in the trap interaction to present a sizable change in the critical parameter $k$; in the perspective of a partial explanation of the observed experimental deviations from the previous theoretical estimates. The present results show that only with a deviation of about $10 \%$ from the harmonic potential, at distances of the order of the oscillator length, it becomes possible to explain theoretically the experimental results reported in Ref. [5], within the error bars. However, this is a quite large strength for the anharmonic term that is not supported by the detailed analysis and description of the experiments given in Ref. [10].

In a more realistic perspective, our present work is predicting the quantitative effect in the critical number of atoms due to anharmonicities that can occur in a trap. For the nonharmonic terms, we have considered the cubic or quartic ones. In cases of other shapes for the anharmonic terms, one can also make qualitative predictions based on the present results. We should point out that our study is not restricted to the example (trap frequencies and symmetry) that we have used; it can be easily extended to other trap arrangements, and can be useful in experimental analysis of condensates with attractive two-body interactions. In this respect, we should note that it has been studied and reported the possible occurrence of anharmonicities in time orbiting potential traps [11]. Our work is considering realistic situations that are close to the actual experiments. So our aim in the present work was to report on the effect of a deviation in the harmonic trap, which increases as we go to regions outside the center of the system.

\section{ACKNOWLEDGMENTS}

We would like to thank Professor Rajat K. Bhaduri for useful discussions and suggestions, and Dr. Elizabeth Donley for pointing us to Ref. [10]. This work was partially supported by Fundação de Amparo à Pesquisa do Estado de São Paulo (FAPESP). L.T. also thanks the Conselho Nacional de Desenvolvimento Científico e Tecnológico (CNPq) of Brazil for support.
[1] M.H. Anderson, J.R. Ensher, M.R. Matthews, C.E. Wieman, and E.A. Cornell, Science 269, 198 (1995).

[2] C.C. Bradley, C.A. Sackett, J.J. Tollett, and R.G. Hulet, Phys. Rev. Lett. 75, 1687 (1995); C.C. Bradley, C.A. Sackett, and R.G. Hulet, ibid. 78, 985 (1997); C.C. Bradley, C.A. Sackett, J.J. Tollett, and R.G. Hulet, ibid. 79, 1170 (1997).
[3] K.B. Davis, M.-O. Mewes, M.R. Andrews, N.J. van Druten, D.S. Durfee, D.M. Kurn, and W. Ketterle, Phys. Rev. Lett. 75, 3969 (1995); M.R. Andrews, M.-O. Mewes, N.J. van Druten, D.S. Durfee, D.M. Kurn, and W. Ketterle, Science 273, 84 (1996); M.-O. Mewes, M.R. Andrews, N.J. van Druten, D.M. Kurn, D.S. Durfee, and W. Ketterle, Phys. Rev. Lett. 77, 416 
(1996).

[4] D.G. Fried, T.C. Killian, L. Willmann, D. Landhuis, S.C. Moss, D. Kleppner, and T.J. Greytak, Phys. Rev. Lett. 81, 3811 (1998).

[5] J.L. Roberts, N.R. Claussen, S.L. Cornish, E.A. Donley, E.A. Cornell, and C.E. Wieman, Phys. Rev. Lett. 86, 4211 (2001); E. Donley et al., Nature (London) 412, 295 (2001).

[6] P.A. Ruprecht, M.J. Holland, K. Burnett, and M. Edwards, Phys. Rev. A 51, 4704 (1995); M. Houbiers and H.T.C. Stoof, ibid. 54, 5055 (1996); R.J. Dodd et al., ibid. 54, 661 (1996); N. Akhmediev, M.P. Das, and A.V. Vagov, Int. J. Mod. Phys. B 13, 625 (1999); L. Bergé, T.J. Alexander, and Y.S. Kivshar, Phys. Rev. A 62, 023607 (2000); C. Huepe, S. Métens, G. Dewel, P. Borckmans, and M.E. Brachet, Phys. Rev. Lett. 82,
1616 (1999); A. Gammal, T. Frederico, and L. Tomio, Phys. Rev. E 60, 2421 (1999).

[7] A. Gammal, T. Frederico, and L. Tomio, Phys. Rev. A 64, 055602 (2001).

[8] A. Gammal, T. Frederico, L. Tomio, and P. Chomaz, J. Phys. B 33, 4053 (2000).

[9] S. Inouye et al., Nature (London) 392, 151 (1998); Ph. Courteille, R.S. Freeland, D.J. Heinzen, F.A. van Abeelen, and B.J. Verhaar, Phys. Rev. Lett. 81, 69 (1998).

[10] J.L. Roberts, Ph.D. thesis, University of Colorado, 2001.

[11] V.G. Minogin, J.A. Richmond, and G.I. Opat, Phys. Rev. A 58, 3138 (1998).

[12] F. Dalfovo, S. Giorgini, L.P. Pitaevskii, and S. Stringari, Rev. Mod. Phys. 71, 463 (1999). 